\title{
Measuring the experience of algorithmic thought digital analogue design in architecture teaching
}

\author{
Laline Elisangela Cenci \\ Universidade Federal de Santa Maria Campus Cachoeira do Sul | Brasil | \\ laline.cenci@ufsm.br
}

of the Iberoamerican Society

of Digital Graphics

Júlio César Pinheiro Pires

Universidade Federal de Santa Maria Campus Cachoeira do Sul | Brasil | julio.pires@ufsm.br

Stéphane Soares Vieira

Universidade Federal de Santa Maria Campus Cachoeira do Sul | Brasil |

soaresvieirastephane@gmail.com

\begin{abstract}
Due to constant technological developments, society's priorities and cultural perspectives have changed, requiring a redefinition of experiences in education. In the field of architecture teaching, the transition from CAD (Computer-Aided Design) to the design systems in other digital media, such as the parametric design, can be observed. This article aims to demonstrate two analog-digital experiences in an architecture school. The methodology consisted of dividing the activities into three stages: analog, logical, and digital. The results are described through quantitative and qualitative data acquired in the experiences. The data allowed toreflect on the strategies adopted, lessons learned, and futures challenges.
\end{abstract}

Keywords: Teaching-learning; Parametric Design; Design Script; Dynamo Studio.

\section{INTRODUÇÃO}

A disseminação do uso de plataformas paramétricas tem possibilitado uma mudança cultural no campo arquitetônico. A transição de sistemas CAD (ComputerAided Design) para sistemas de design em outros meios computacionais, como por exemplo, os ambientes de programação visual para modelagem paramétrica, gera consequências diretas no uso do computador como ferramenta que colabora no processo criativo (Celani\& Vaz, 2011). O desenvolvimento de projetos em plataformas paramétricas permite gerar formas mais complexas e essas plataformas integram o processo de simulação, avaliação e fabricação, ao alcance do projetista (Oxman, 2017). O emprego dessas plataformas requer um domínio de novos meios de interação relacionados à modelagem tridimensional (3D). No âmbito digital, pressupõe o uso de diferentes interfaces: a representacional, a paramétrica e a algorítmica, pois, cabe ressaltar que o processo de projeto em arquitetura possui a necessidade de modificações constante. Segundo Bueno (2018), o projeto arquitetônico é marcado por quatro tipo de representações: croquis, modelos $3 \mathrm{D}$, análise de desempenho e desenhos técnicos. Cada tipo de representação possui uma ferramenta associada, uma do grupo de mídia física (como o papel) e outra do grupo de mídia digital. Durante o processo de projeto existe uma integração do meio físico (analógico) com o digital.

A transferência da representação física para a digital, geralmente feita através de sistemas CAD, vem sofrendo alterações com a disponibilidade de aplicativos paramétricos (Alves, 2009). No âmbito da academia, no processo de projeto, é necessário que ocorra a preparação dos estudantes para a implantação desse sistema através da inserção do pensamento lógico do desenho paramétrico, que também requer o desenvolvimento do pensamento matemático. Pensar matematicamente refere-se a teoremas e construções usadas para definir a linguagem script da representação e geração do design (Oxman\&Gu, 2015). No presente artigo serão apresentadas duas experimentações didática de integração do analógico-digital onde desenvolveu-se objetos através de um método que vai desde a concepção inicial considerada analógica até a modelagem algorítmica, levando em conta recursos de design script. A modelagem geométrica através de design script demanda uma ampliação de habilidades cognitivas e computacionais para entender a formalização, representação e a linguagem em código dos processos de criação (Aish, 2011). Isso vai além do conhecimento arquitetônico e requer capacitação com foco em atividades que instiguem o pensamento lógico e gerem conhecimentos mais profundos em relação ao software paramétrico utilizado.

Oxman (2017), sugere um reexame das teorias e metodologias para guiar pesquisas e desenvolvimentos futuros, levando em consideração a importância da experimentação para processamento de informações e absorção de conhecimento e a evolução do projeto digital como um campo de conhecimento.

Portanto as experiências didáticas relatadas neste trabalho objetivaram, além de apresentar um novo conhecimento técnico para os estudantes, instigá-los a analisar criticamente as decisões projetuais, expandir a imaginação e investigar as especificidades dos processos 
experimentais e geração de alternativas, para que novas propostas didáticas possam ser consideradas e inseridas no ensino de desenho digital em arquitetura.

\section{O PROCESSO DE PROJETO E AS DIFERENTES INTERFACES}

De acordo com Bueno (2018), o processo de projeto possui uma progressão de etapas. Estas etapas são marcadas pelos diferentes tipos de representações do desenho de projeto e, como tais, podem ser feitas através de diferentes ferramentas de acordo com duas interfaces básicas, a digital e a analógica. Para as etapas iniciais, a concepção das ideias é feita à mão, através dos croquis, que servem como instrumento de busca, investigação e experimentação (Romcy, 2017).

$\mathrm{O}$ ato de desenhar durante o processo de projeto é uma forma do projetista estabelecer um meio de comunicação daquilo que está imaginando para o projeto em questão. Em relação ao ensino de projeto, Florio (2011A) afirma que existem diferentes métodos e processos que servem para auxiliar na condensação dos conhecimentos dos estudantes em cada fase de projeto. Por isso, é importante que os estudantes saibam a intenção e a utilidade de cada uma das ferramentas, sejam elas: esboços, desenhos técnicos, maquetes físicas ou modelos digitais (Florio, 2011A). Os modelos físicos podem ser utilizados para atividade de criação e também para a visualização plástico-espacial da ideia, que permite realizar estudos tridimensionais (Romcy, 2017). A alternância entre modelos físicos e desenhos permite a compreensão das relações espaciais e possibilita melhores condições de avaliação sobre o que se produz a cada momento (Florio, 2011A). Já em relação às interfaces digitais, de acordo com Hudson (2010), a utilização de modelo paramétrico durante as diversas fases projetuais, possibilitaria o aumento da eficiência, reduzindo as cargas de trabalho manual. A modelagem paramétrica permite relacionar critérios de decisões de projeto com um modelo de informação, alterar parâmetros e gerar simulações e análise de alternativas, além de possibilitar a produção de diversas versões de um mesmo modelo (Romcy\& Tinoco, 2015).

A linguagem analógica caracteriza-se por ser mais ambígua e imprecisa, por estar relacionada às relações, artes, subjetividades e também costuma ser utilizada para definir uma ligação com o jeito de ser, emoções, afetos. Já a linguagem digital, é mais precisa e abstrata, ligada ao conhecimento científico e tecnológico. As duas linguagens possuem limitações, por isso, combinam-se as duas de forma a emitir ou receber, traduzindo constantemente uma mensagem analógica para a digital e vice-versa. Dessa maneira, é necessário estabelecer a comunicação com os computadores, definir comandos que os permitam interpretar e realizar as operações que irão resultar na resposta, como por exemplo uma geometria. Considera-se o desenho como uma linguagem de comunicação entre analógico e digital, onde se destaca a importância desse processo de troca, pois se for descuidado, é possível que se crie uma barreira comunicativa entre esses dois meios, principalmente tratando de projetos (Rheingantz, 2016).

\section{DESENHO, EXPRESSÃO E CRIATIVIDADE}

A criatividade é um ponto chave no ensino de projeto em arquitetura e é definida pela capacidade de produzir algo novo ou adaptado no contexto em que se torna necessário, por exemplo, desenvolver solução para determinado projeto (Florio, 2011B). O desenho à mão é uma forma dos estudantes estabelecerem uma conexão entre o mental e o visual, transmitindo suas ideias para o meio físico. Esse processo permite que sejam feitas reflexões, alterações e modificações de forma rápida, criando uma conversação entre a mente e o desenho em elaboração (Lawson, 2005).

Atualmente a intensa presença do processo tecnológico, como a indústria do entretenimento e a abundância dos meios de comunicação visual, faz com que as pessoas sejam bombardeadas por diversas imagens e estímulos. A mudança rápida de ferramentas, somada a efemeridade, faz com que a percepção do espaço seja cada vez mais superficial, através de um olhar rápido e desatento. 0 exercício de percepção deve ser trabalhado de modo contínuo, com o objetivo de desenvolver o olhar. O desenho analógico, por exemplo, é uma ferramenta para esse tipo de exercício, à medida que o desenho é desenvolvido, o olhar e a percepção espacial também são (Vizioli \& Pacheco, 2013).

Segundo Ching (2011), o desenho à mão livre é o meio mais intuitivo para registrar graficamente observações, ideias e experiências, devido à sua natureza tátil e cinestésica. A integração dos processos analógicos envolvendo elementos gráficos e físicos com os processos digitais tem sido algo cada vez mais desejável no ensino de projeto, pois é fundamental que o aluno tome consciência do processo de investigação a ser empregado para que consiga entender a ideia que está imaginando, facilitando a elaboração e a transmissão da proposta. (Florio, 2011A).

Além disso, a cognição, quando relacionada com processo de projeto, envolve o entendimento do problema geral do projeto. $\mathrm{O}$ processamento de informações, percepção e raciocínio, são gerados através de símbolos, tais como esboços, desenhos, textos, modelos físicos e digitais (Florio, 2011A). É preciso exercitar o raciocínio lógico para desenvolver a habilidade de reconhecimento e manipulação de geometrias para aprimorar a criatividade durante o processo de projeto. Por isso a alternância de métodos como desenhos e modelos físicos permite que os estudantes compreendam relações espaciais e forneça melhores condições para as tomadas de decisões (Florio, 2011A).

\section{INTERFACE DIGITAL}

Diversas mudanças ocorreram na prática de projeto e no processo de ensino-aprendizado de arquitetura devido a difusão do uso da tecnologia computacional. Segundo Henriques (2016), nos anos 80, o computador foi introduzido na indústria e no ensino com a pretensão de substituir o lápis. Dessa maneira o computador era utilizado na fase final de projeto, para "passar à limpo" o desenho 2D feito à lápis. Porém, com a evolução dos meios tecnológicos, os softwares passaram a ser utilizados como forma de gerar modelos tridimensionais, que permitem a visualização de cada modificação no projeto, auxiliando na melhoria da percepção espacial.

Segundo Kasik, Buxton e Ferguson (2005), em seu manifesto Ten CAD Challenges, que lista os 10 desafios dos sistemas CAD, o quarto desafio diz respeito à possibilidade de realização de simulações durante o processo de projeto, pois isso permitirá considerar o comportamento dos modelos e não somente a forma. Os mesmos autores citam, no desafio 7 , as possibilidades que os sistemas de modelagem 3D trouxeram no final da década de 1980, permitindo gerar o modelo inteiro de um 
avião comercial, por exemplo. Deste modo a utilização de softwares que permitem manipulação de informações e parâmetros, ou seja, plataformas paramétricas, podem aumentar a flexibilidade no processo de projeto e a criação de formas mais complexas, além de gerar uma grande quantidade de dados e informações.

$\mathrm{Na}$ área da arquitetura não existe uma única solução para um mesmo problema de projeto. Mesmo que sejam identificadas as mesmas condicionantes e restrições devido ao contexto projetual, cada arquiteto com sua visão, conhecimento e experiências anteriores, imaginará inúmeras possibilidades que levarão ao caminho de uma solução (Florio, 2011A).

Através do processo paramétrico de projeto, no contexto digital, desenvolve-se um modelo ou descrição de um problema em que a representação é baseada nas relações entre objetos controlados por parâmetros. Apesar dos modelos paramétricos serem dinâmicos e de rápida resposta para problemas específicos, eles consistem em estruturas complexas que demandam tempo para elaboração. Como se fundamentam em relações entre objetos, os processos paramétricos de projeto são dependentes das definições dessas relações e da habilidade do projetista (Woodbury, 2010).

Woodbury (2010) destaca ainda que existem certas habilidades que devem ser desenvolvidas para que se consiga trabalhar efetivamente com o sistema de modelagem paramétrica. Algumas das habilidades já são conhecidas, outras novas, a primeira delas é a concepção de fluxo de dados. É necessário saber interagir com os dados do modelo paramétrico, como conceber, editar e organizar pois, isso traz consequências diretas nas possibilidades de projeto. A segunda habilidade, denominada pelo autor "dividir para conquistar", consiste dividir o projeto em partes, projetar cada parte e garantir a interação entre elas durante o processo, criando também relações claras e compreensiveis para que os dados possam fluir adequadamente entre as diferentes partes do projeto.

Como o processo de modelagem paramétrica consiste em projetar várias partes que compõem um todo, é imprescindível que se desenvolvam a habilidade de nomear as partes, dessa forma organizando melhor o fluxo de projeto. Também é necessário o desenvolvimento do pensamento abstrato, tornando o modelo aplicável em diversas situações, com caráter mais genérico, excluindo termos muito específicos, para que uma parte de um projeto ou modelo possa ser reutilizada em outro e assim por diante. Além disso existem elementos fundamentais da matemática, como por exemplo as declarações condicionais, os operadores lógicos, as funções e etc. para dominar a modelagem paramétrica. $\mathrm{O}$ desenvolvimento desse pensamento auxilia a criar novas estratégias projetuais

De acordo com Romcy et al. (2015), a partir de manipulações dos parâmetros que caracterizam o modelo é possível gerar inúmeras variações de um projeto, enriquecendo as operações projetuais que a modelagem paramétrica proporciona. A mesma autora afirma ainda que, diante das novas possibilidades que extrapolam descrições gráficas de representação e visualização, tornou-se necessária a reflexão sobre as especificidades da abordagem paramétrica para sua inserção no ensino de projeto, considerando novas propostas didáticas.
Diante do risco de construir uma barreira comunicativa decorrente da crescente dependência de uma lógica digital, argumenta-se em favor da necessidade de enfatizar a importância do desenho como linguagem de comunicação analógica e digital causada pelas demandas crescentes de precisão e eficiência, cujo preço pode vir a ser abrir mão de características mais humanas (Rheingantz, 2016).

Segundo Rheingantz (2016), existe um dilema sobre o uso do computador como ferramenta de projeto, pois a disseminação dessa ferramenta reflete no ensino de projeto de arquitetura e como consequência a tendência crescente de substituir a tecnologia analógica pela digital no processo de projeto. Destaca-se que ocorre a mistura dos processos e produtos, analógico e digital, e não a exclusão de um ou outro.

Celani (2008) relata que as disciplinas inseridas no currículo obrigatório ainda não são suficientes para causar um impacto na maneira de ensinar a projetar e que apesar dos esforços, ainda existem muitos cursos que oferecem o ensino de informática de forma retrógrada e com o objetivo de oferecer experiência para a demanda do mercado de mão de obra, não cultivando o pensamento crítico. Por isso, a importância de pesquisas em áreas de tecnologias para auxiliar no processo de projeto e garantir o ensino de qualidade durante a formação acadêmica.

Diante desse quadro, para a integração do processo analógico e digital e para o desenvolvimento de conhecimentos específicos de modelagem paramétrica, do ponto de vista do ensino e aprendizagem, foram propostos dois experimentos no ano de $2019 \mathrm{com}$ estudantes de arquitetura e urbanismo, de acordo com a metodologia a seguir.

\section{METODOLOGIA}

Durante os dois semestres de 2019 foram realizadas duas experiências de ensino-aprendizagem de modelagem paramétrica. $\mathrm{Na}$ Experiência 1 foi proposto 0 desenvolvimento de um mobiliário residencial e na Experiência 2 foi proposto o desenvolvimento de um pavilhão.

A metodologia utilizada para as experiências foi dividida em três etapas, conforme o diagrama da Figura 1.

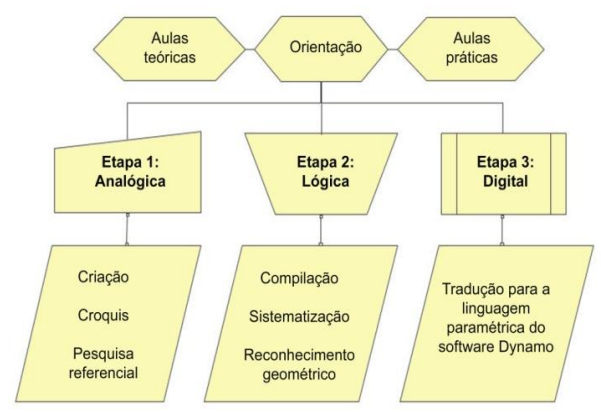

Figura 1: Diagrama Metodológico das Experiências Didáticas. Fonte: Elaboração própria. 
A plataforma escolhida para o desenvolvimento das experiências foi o Autodesk Dynamo Studio $\AA$, que possui licença estudantil e estava disponível no laboratório onde as atividades foram realizadas.

Inicialmente ocorreu o processo de preparação através de aulas teóricas, aulas práticas e orientações. Durante a preparação, foi introduzido o pensamento lógico e conhecimentos em design script, além da demonstração da interface do software utilizado.

Seguindo o diagrama metodológico, foram propostas três etapas: analógica, lógica e digital. Na etapa analógica fo realizado um processo de geração de dados manualmente. Esses dados são representados pela criação e expressão através de croquis relativos à cada experiência e também pela pesquisa referencial gerada pelos estudantes. Como ferramenta, foi utilizado papel, lápis e borracha e em alguns casos, os estudantes fizeram uma maquete volumétrica de estudo com dobradura de papel.

Durante a segunda etapa, a lógica, após o objeto já estar brevemente definido através dos desenhos à mão, os alunos realizaram a análise de seus croquis e a sistematização do desenho. Para isso, foram feitas operações manuais através da descrição lógica das geometrias e também a criação de diagramas.

$\mathrm{Na}$ terceira etapa, a digital, os estudantes utilizaram como base as operações manuais da etapa anterior para a criação dos algoritmos no software, ou seja, a tradução da descrição lógica da forma para rotinas de scripts. Os alunos interagiram com o software e geraram objetos utilizando programação visual com o método design script. Essa etapa contou com o acompanhamento dos professores que auxiliaram na criação e adaptação dos algoritmos caso a caso.

\section{EXPERIÊNCIA 1}

Seguindo a metodologia apresentada, a experiência 1 ocorreu conforme as três etapas descritas. Durante a preparação para a atividade, foi realizada a modelagem de um mobiliário de exemplo como forma de os alunos experimentarem a criação de scripts, além de iniciar o processo da inserção do pensamento lógico. No decorrer das aulas expositivas, foi apresentada a interface do software e, durante as aulas práticas, foi desenvolvida a modelagem de uma mesa, que os estudantes acompanharam e fizeram o passo a passo junto com os professores orientadores.

O mobiliário proposto para a Experiência 1 foi uma cadeira, e para iniciar o processo de criação, foi solicitado aos alunos que fizessem croquis à mão para expressar as primeiras ideias. Dessa forma, os alunos puderam expor de maneira rápida e flexível o que estavam imaginando para a forma da cadeira, podendo realizar modificações e gerar reflexões acerca do que estava no papel. Após preestabelecer uma forma, sugeriu-se que os alunos pesquisassem imagens de referências de outras cadeiras que tivessem semelhança com as que foram desenhadas. Com a pesquisa referencial os alunos puderam observar como cada parte de uma cadeira se relacionam com a sua forma geral. Em alguns casos, os alunos encontraram inspiração em cadeiras icônicas, como por exemplo a cadeira Vermelha e Azul, que foi desenhada por Gerrit Rietveld e fez parte do movimento De Stijl. Alguns croquis elaborados pelos estudantes na etapa analógica podem ser observados na Figura 2

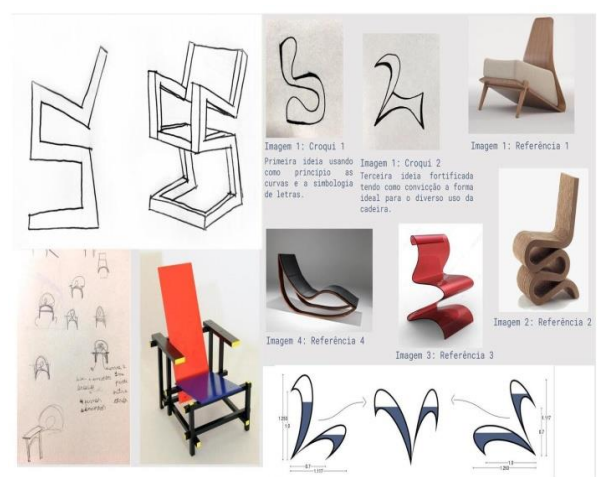

Figura 2: Croquis feitos pelos estudantes durante a etapa analógica e imagens de referência de cadeiras icônicas. Fonte: Autores.

$\mathrm{Na}$ segunda etapa, foi solicitado aos estudantes que descrevessem os seus desenhos em geometrias, de forma a compreender quais elementos estavam compondo o desenho e como fazer cada parte. Para isso, os estudantes fizeram a descrição lógica da forma e desenvolveram uma espécie de roteiro, um passo a passo para inserir a geometria no software. Foi necessário entender quais partes estavam compondo a cadeira e como localizar a geometria de cada elemento, sendo eles: pernas, braços, assento e encosto. O foco dessa etapa foi pensar racionalmente no modelo e nas partes de elaboração, principalmente na descrição de cada ponto e suas respectivas coordenadas através do plano ortogonal de referência, pois estes elementos foram inseridos na plataforma para gerar o mobiliário. Foi necessário pensar também na localização de cada peça em relação à origem e em relação às outras peças, para que se encaixassem. $\mathrm{O}$ desafio foi entender as partes em relação ao todo para transformar em uma descrição lógica.

Na etapa digital os alunos utilizaram como ferramenta base os desenhos e o roteiro descritivo que criaram e também os algoritmos aprendidos através do acompanhamento da modelagem da mesa. Os estudantes traduziram as descrições lógicas para algoritmos e de acordo com a necessidade de cada trabalho, utilizaram e adaptaram os algoritmos aprendidos durante a preparação.

A modelagem algoritmica, para todos os alunos, iniciou com a inserção de pontos. No método design script do Dynamo Studio geometrias são criadas a partir da inserção de nós. O processo de modelagem se dá pela ligação lógica entre os nós (Figura 3).

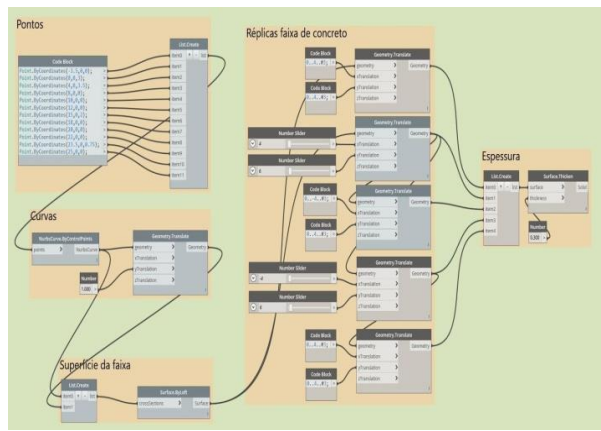


Figura 3: Rotinas de scripts criadas durante a etapa digital. Fonte: Autores.

Os nós podem representar geometrias, valores numéricos, transformações, listas, funções, atributos, instâncias e etc. Cada trabalho teve um desenvolvimento diferente, gerando um conjunto de nós e parâmetros específicos. Mesmo que os algoritmos utilizados tenham sido similares para alguns alunos, a ordem em que foram inseridos os nós e criadas suas conexões resultaram em geometrias completamente diferentes.

\section{RESULTADOS DA EXPERIÊNCIA 1}

Ao fim de cada experiência, os estudantes produziram um trabalho final em formato de banner, composto por introdução, desenvolvimento, resultados e discussões. Nos banners os estudantes descreveram a experiência de interação com software de modelagem paramétrica, apontando algumas dificuldades e também potencialidades encontradas na atividade proposta. A Figura 4 apresenta dois banners como resultados da metodologia aplicada.
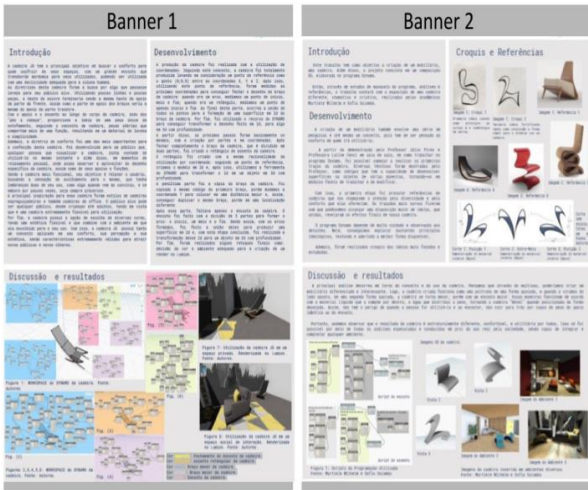

Figura 4: Dois exemplos de banners produzidos pelos estudantes como resultado da Experiência 1. Fonte: Autores.

Destaca-se, pelo resultado dos trabalhos dos estudantes, que mesmo utilizando como base as mesmas aulas teóricas e algoritmos de referência, as cadeiras foram criadas de maneira diferente e com geometrias distintas. Cada estudante buscou alternativas de acordo com a complexidade do objeto que estava propondo e também as peculiaridades da geometria. Ressalta-se a importância do desenvolvimento da autonomia dos estudantes e de instigar o pensamento criativo, lógico e matemático, pois com uma base de aulas teóricas, breve explanação do software e a apresentação de um modelo simplificado de objeto, os estudantes conseguiram elaborar a geometria de uma cadeira, conforme os seus croquis iniciais e sua descrição lógica. Atingiu-se o resultado esperado em relação à compreensão de um nível básico do software utilizado, ao pensamento lógico proposto e aos conhecimentos técnicos apresentados sobre programação com uso de design script. Contudo, durante a descrição lógica dos objetos (etapa 2 do diagrama metodológico), não foi solicitada a apresentação de cada roteiro criado pelos estudantes. Dessa forma os mesmos permaneceram como rascunhos e apontamentos nos croquis, impossibilitando a exposição de material referente a essa etapa. Sendo assim, a formalização da descrição lógica da geometria através de diagramas foi aprimorada na segunda Experiência.

\section{EXPERIÊNCIA 2}

Na Experiência 2 foi proposta a criação de um pavilhão com algumas características previamente definidas pelos docentes. Durante a preparação, foi apresentada a interface do software e realizados exercícios práticos de criação de pontos, de superfícies e de sólidos. Esses exercícios serviram de experimentação para introdução do pensamento lógico necessário para realização da atividade.

Na primeira etapa (analógica) foi proposto aprimoramento da criatividade e expressão através de croquis à mão. Em alguns casos os estudantes propuseram maquetes físicas feitas de dobradura de papel como forma de estudos volumétricos, que podem ser observados na Figura 5. Também foi feita a pesquisa referencial de modelos similares, inspirando os alunos a imaginarem um contexto de inserção para o pavilhão proposto.

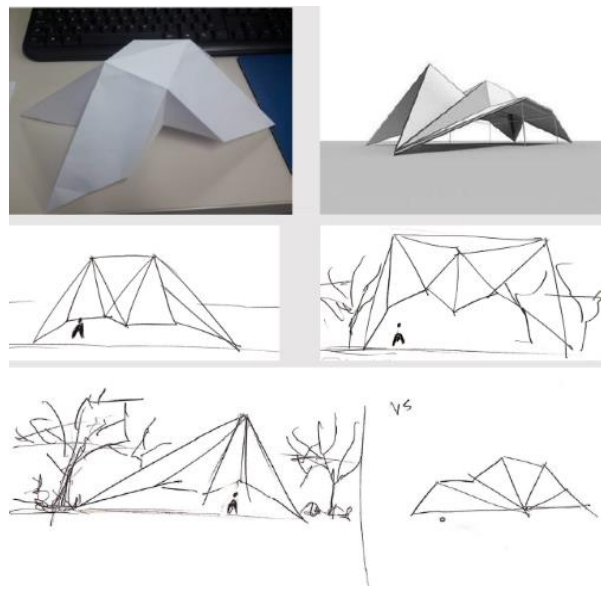

Figura 5: Maquete volumétrica e croquis elaborados pelos estudantes durante a etapa analógica. Fonte: Autores.

Após a finalização do estudo formal do pavilhão, com um desenho preestabelecido, os estudantes utilizaram recurso gráfico para criação de diagramas que foram responsáveis por auxiliar no entendimento da lógica da forma. Em um exemplo de diagrama proposto durante esta parte da atividade, fica claro que os estudantes dividiram a geometria em elementos e descreveram os parâmetros de cada um, como percebe-se na Figura 6. 


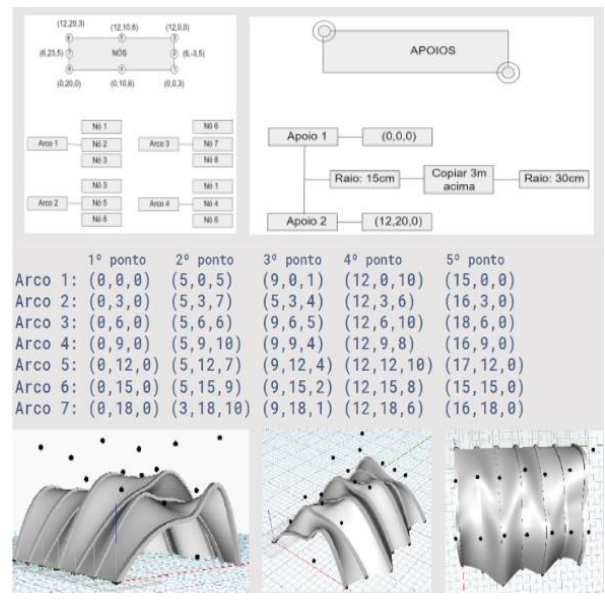

Figura 6: Diagramas realizados pelos estudantes durante a etapa lógica. Fonte: Autores.

Da mesma forma como ocorreu na Experiência 1, nesta etapa os alunos utilizaram como base o processo da descrição lógica e os diagramas da forma do pavilhão. Este processo auxiliou na criação das rotinas de scripts. Os algoritmos criados foram adaptados através da orientação dos professores que fizeram o acompanhamento dos trabalhos durante o desenvolvimento dessa etapa, conforme a necessidade de cada um. Em alguns casos, os estudantes organizaram os scripts por partes, como pode ser observado na Figura 7. Também nomearam alguns conjuntos de nós criando pequenos blocos, organizando os scripts na interface do Dynamo Studio. Isto auxiliou no entendimento das interações entre os parâmetros e também tornou mais fácil encontrá-los quando era necessário fazer modificações.

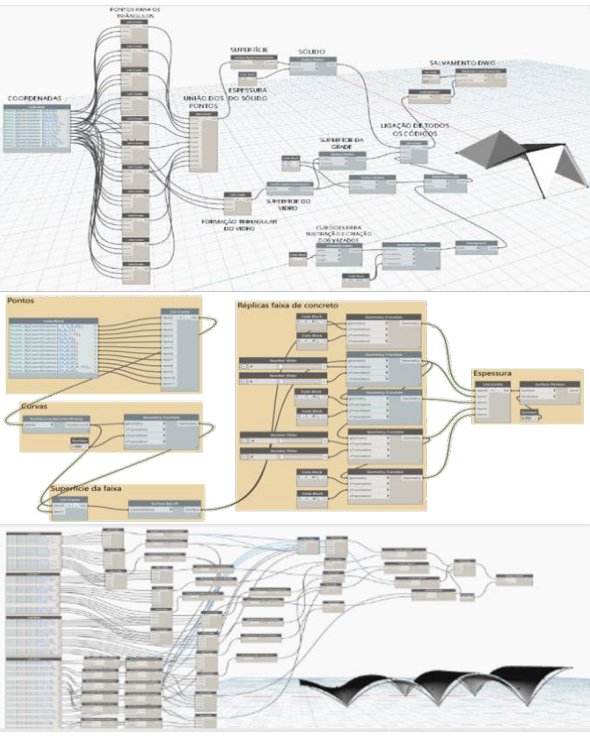

Figura 7: Rotinas de scripts criadas pelos estudantes durante a etapa digital. Fonte: Autores.

\section{RESULTADOS DA EXPERIÊNCIA 2}

Nesta experiência, como na experiência 1, os estudantes criaram banners em que descreveram a atividade realizada através da estrutura proposta pelos docentes: introdução, desenvolvimento, discussão e resultados. Porém, adicionou-se os diagramas aprimorados na etapa lógica.

Também nesta segunda experiência foi proposto o desenvolvimento de uma rotina para realização da impressão 3D dos modelos gerados. A rotina iniciou na preparação e exportação do modelo no formato DWG e posteriores ajustes no software AutoCAD versão educacional (também disponível no laboratório). Após ajustes, foram gerados arquivos do tipo STL (StereoLithography). Estes arquivos foram inseridos no software CURA, próprio para impressão 3D. A experiência finalizou nesta etapa e nos banners de apresentação dos alunos constaram os passos descrevendo todo este processo. A Figura 8 apresenta dois exemplos de banners produzidos durante a experiência 2 .

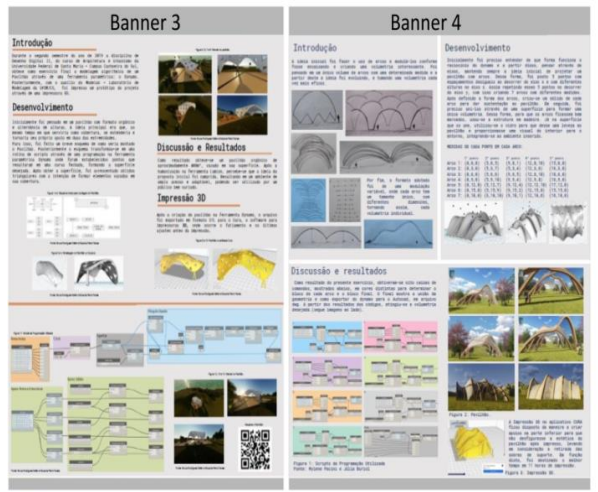

Figura 8: Exemplo de dois banners resultantes da experiência 2. Fonte: Autores.

\section{DISCUSSÃO}

Para avaliação das experiências realizadas no ano de 2019 aplicou-se um questionário de pesquisa de satisfação aos estudantes que participaram destas duas experiências. De acordo com as respostas, $66,7 \%$ ficaram muito satisfeitos em relação a etapa analógica, 58,3\% demonstram-se muito satisfeitos com a etapa lógica e apenas $41,7 \%$ ficaram muito satisfeitos com a etapa digital. Além das respostas, os estudantes escreveram um comentário para cada etapa, auxiliando na melhor avaliação e na coleta de sugestões para as atividades futuras.

De acordo com os comentários, em relação a etapa analógica, demonstraram que produzir croquis e pesquisar por referências auxiliou no desenvolvimento criativo e também como forma de marcar um ponto de partida para a modelagem. Alguns comentários sugeriram enfatizar mais essa etapa para que as geometrias mais complexas pudessem ser melhor estruturadas. Em relação à etapa lógica, os estudantes demonstraram satisfação por aprender uma técnica nova, que parecia ser complexa e ficou clara durante a etapa da elaboração dos diagramas. Relataram também que apesar de parecer um quebra- 
cabeça, essa etapa é muito importante para estruturar o raciocínio que deve ser utilizado para criar algoritmos no software Dynamo. Destacou-se a dificuldade de descrever a forma através de um passo a passo, pois foi necessário realizar a desconstrução da geometria para entender as partes que estavam compondo o todo, mas que é de extrema importância para a etapa digital. Constatou-se que para etapa digital, houve mais apontamentos negativos em relação às etapas anteriores, demonstrando dificuldade de manipular o software escolhido para as atividades. Alguns estudantes relataram que durante a experiência ficaram limitados em relação aos algoritmos aprendidos em aula e enfrentaram dificuldade de encontrar tutoriais do software na internet. Porém pensam que é muito positiva a facilidade de modificar os parâmetros, como ajustar volumes e alterar alturas. Além disso, constatou-se que a dificuldade dos estudantes dependia da complexidade da geometria que foi proposta e isso necessitava mais o auxílio dos orientadores. As duas experiências exigiram dos estudantes empenho para compreender e estruturar um raciocínio lógico para a modelagem paramétrica.

A medida em que as experiências aconteceram, destacase que os resultados coletados nos questionários sobre as etapas para o exercício de pensamento algorítmico apontaram um paralelo entre o que MORIN ${ }^{1}$ apresenta sobre duas formas de compreensão: uma intelectual onde a explicação seria suficiente para o conhecimento de algo; e a outra é a compreensão humana intersubjetiva que va além da explicação. Neste sentido, existem obstáculos intrínsecos às duas compreensões, não somente quando do uso do desenho analógico, mas também a dificuldade explicitada na aversão àquilo que não é familiar, como a inserção de códigos e algoritmos no processo de desenvolvimento de projeto.

\section{CONCLUSÕES}

De caráter desafiador, as atividades instigaram o desenvolvimento criativo e a autonomia para que os estudantes explorassem mais sobre a modelagem através de scripts, além da melhora da postura crítica em relação ao que cada um produziu. O desafio para o futuro é compreender que o desenho pode ser um elemento transformador no processo de projeto, não só em relação aos modos analógico ou digital, mas em um contexto propositivo onde a complexidade da forma deixa de ser uma barreira e o conjunto de dados gerados no processo possa ser gerenciado com mais eficiência.

As experiências relatadas destinaram-se não apenas em difundir os aspectos técnicos de um software, mas promover uma mudança de postura do acadêmico ao desenvolver sua capacidade crítica para análise de projetos, além de expandir sua imaginação em processos criativos.

A promoção do acréscimo de competências relacionadas à atividade do arquiteto deve ser sempre fomentada na academia. O rápido desenvolvimento das ferramentas paramétricas de auxílio ao projeto não pode ser confundido com uma substituição de métodos. Sistemas representativos mais tradicionais permanecerão sendo utilizados. Entretanto deve-se ter em conta que a escolha do método é tão ou mais importante para se alcançar melhores resultados dentro do processo de projeto em arquitetura.

\section{AGRADECIMENTOS}

Os autores agradecem aos estudantes que participaram das atividades didáticas descritas neste trabalho.

\section{REFERÊNCIAS}

Aish, R. (2011). DesignScript: Origins, Explanation,Illustration. Computational Design Modelling, 1-8.

Alves, G.M. (2009). O desenho analógico e o desenho digital: a representação do projeto arquitetônico influenciado pelo uso do computador e as possíveis mudanças no processo projetivo em arquitetura. Dissertação de mestrado, Universidade Federal do Mato Grosso do Sul, Campo Grande, MS, Brasil.

Bueno, E. (2018). Interação entre o arquiteto e as ferramentas digitais nas etapas iniciais de projeto. Revista Prumo (5).

Celani, M.G. \& Vaz, C.E. (2011). Scripts em CAD e ambientes de programação visual para modelagem paramétrica: uma comparação do ponto de vista pedagógico. Proarq, 18(1), 117-194.

Celani, M.G. (2008). A importância da pesquisa na formação de docentes: o caso da "informática aplicada à arquitetura e urbanismo". Cadernos de Pós Graduação em Arquitetura e Urbanismo, v.7, n.1.

Ching, F.D.K. (2011). Representação gráfica em arquitetura. $5^{\text {a }}$ ed. Porto Alegre: Ed. Bookman.

Florio, W. (2011A). Análise do processo de projeto sob a teoria cognitiva: sete dificuldades no atelier. Arquiteturarevista, 7(2), 161-171.

Florio, W.(2011B). Modelagem paramétrica, criatividade e projeto: duas experiências com estudantes de arquitetura. Gestão\& Tecnologia de Projetos, 6(2), 43-66.

Henriques, G. (2016). Arquitetura algorítmica: técnicas, processos e fundamentos. Anais IV ENANPARQ, Sessão 39. Projeto digital e fabricação na arquitetura: ensino, pesquisa e desafio. Organização: Cláudia Costa Cabral, Carlos Eduardo Comas, edição PROPAR/UFRGS, Porto Alegre.

Hudson, R. (2010). Estratégias para o projeto paramétrico em arquitetura: uma aplicação de prática liderou a pesquisa. Tese de doutorado, Universidade de Bath, Departamento de Arquitetura e Engenharia Civil, Inglaterra, Reino Unido.

Jabi, W. (2013). Parametric Design for Architecture. London, UK. Ed: Laurence King Publishing Ltd.

Kasik, D.J. Buxton, W., \& Ferguson, D.R. (2007). Ten CAD challenges. ACM SIGGRAPH 2007 Papers - International Conference on Computer Graphics and Interactive Techniques, 81-92.

Lawson, B. (2005). How designers think. $4^{\text {a }}$ ed. Oxford, UK. Ed: Elsevier.

Morin, E. Os sete saberes necessários à educação do futuro.São Paulo: Cortez, Brasília: UNESCO, 2002.

Oxman, R. (2017). Thinking difference: theories and models of parametric design thinking. Design Studies, 52, 4-39.

Oxman, R. \&Gu, N. (2015). Theories and models of parametric design thinking. Real Time - Proceedings of the $33^{\text {rd }}$ eCAADe Conference Vienna, Austria, 477-482. 
Rheingantz, P. A. Projeto de arquitetura: processo analógico ou digital. Gestão e Tecnologia de Projetos, São Carlos, v. 11, n. 1, p. 95-102, jan./jun. 2016.

Romcy, N.M. (2017). Abordagem paramétrica e ensino de projeto: proposição de diretrizes metodológicas considerando estratégias curriculares e 0 atelier de projeto. Tese de doutorado, Universidade Federal do Rio Grande do Norte, Natal, RN, Brasil.

Romcy, N.M. \& Tinoco, M.B.M. (2015). Reflexões sobre as interfaces entre a modelagem paramétrica e outros processos de representação no projeto de arquitetura. Blucher. 322-327.

Romcy, N.M.; Tinoco, M.B.M.; Cardoso, D.R. (2015) Reflexões sobre a abordagem paramétrica no ensino de projeto. V!RUS, n.11.
Vizioli, S.H.T.; Pacheco, P.R. (2013) O desenho no processo projetivo: estudo das representações gráficas de projetos de Paulo Mendes da Rocha. Seminário Internacional "Representar Brasil 2013" As representações na Arquitetura, Urbanismo e Design, II, São Paulo, SP, Brasil.

Wills, J.E.B. (2014). Uso de tecnologias digitais nas etapas iniciais de projeto: proposição de diretrizes metodológicas, considerando estratégias curriculares e o atelier de projeto. Tese de doutorado, Universidade Federal do Rio grande do Norte, Natal, RN, Brasil.

Woodbury, R. (2010). Elements of parametric design. Routledge. 